\title{
Prothèse adjointe complète : la mise en condition tissulaire est-elle toujours d'actualité ?
}

\section{Complete denture: the tissue conditionner treatment,} is it alwarys a present treatment?

\section{MOTS-CLEFS :}

- Prothèse complète, résine ò prise retardée, mise en condition tissulaire.

\section{KEYWORDS:}

- Complete denture, soft-liner material, soft tissue treatment.
AOS 2014;268:24-28 DOI: $10.1051 / \mathrm{aos} / 2014204$ (C) EDP Sciences 2014

\section{Résumé}

La mise en condition tissulaire est une approche thérapeutique bien connue mais malheureusement pas toujours bien appréhendée. Ses indications sont précises, mais ses contre-indications peu respectées. Celles-ci sont cependant dictées par le principe du consentement éclairé, son non-respect pouvant être à l'origine de conflits entre le praticien et le patient. La bonne réalisation de cette thérapeutique dépend de la parfaite connaissance des caractéristiques des résines ò prise retardée, des caractéristiques des différentes phases physiques et de leur rôle clinique. Cliniquement, les séquences opératoires sont parfaitement codifiées et surtout sont déterminées par la durée des différentes phases physico-chimiques spécifiques aux matériaux utilisés.

\section{Abstract}

The tissue conditioner treatment is a well-known clinical approach, but unfortunately, it is not always grasped.

The indications are precise, but the contraindications are often unrespected or forgotten. In reality, they are induced by the informed consent principle. Its unrespect could be the beginning of legal problems between the patient and the practitioner. The right achievement of the clinical steps depends on the perfect knowledge of soft-liner material, their physical phases and their clinical functions. Clinically, the steps are perfectly codified and, above all, the times dedicated to their uses must be in accordance with the physical characteristics of the used soft-liner materials.

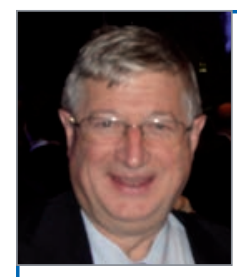

- Olivier HUE, PU-PH,UFR d'Odontologie Université Aix Marseille 27 Bd. Jean Moulin, 13005 Marseille. Service d'Odontologie - Hôpital de la Timone

Marie-Violaine BERTERETCHE, PU-PH, UFR d'Odontologie Garancière Université Paris-Diderot, Paris 7, 5 Rue Garancière, 75006 Paris. Pôle Odontologique Hôpital Rothschild 75012 Paris 
$\mathrm{C}$ hez les patients porteurs de prothèses adjointes complètes, le traitement des surfaces d'appui altérées par le port de celles-ci a toujours été un problème. Pour supprimer ces altérations, blessures, inflammations plus ou moins étendues, diverses solutions thérapeutiques ont été proposées. Au premier rang de celles-ci, la suppression du port des prothèses, mais ce non-port ne permettait pas de rétablir l'intégrité des tissus des surfaces d'appui. De plus, de nos jours, il semble difficile à un praticien de conseiller voire d'imposer à un patient de ne plus porter ses prothèses pendant quelques jours.

Afin de permettre au patient de garder ses prothèses, Nelson, dès 1947, préconisait l'utilisation de la pâte à loxyde de zinc - eugénol. Aux États-Unis, Lyttle (1957), Chase (1961), Pound développèrent l'usage des résines à prise dite retardée $[1,2,3]$. En France, Joseph Lejoyeux (1962) exploita cette technologie et créa l'expression de « mise en condition tissulaire » qu'il définissait ainsi [4] : "On appelle mise en condition, l'ensemble des préparations et thérapeutiques destinées à placer les tissus buccaux dans des conditions idéales pour recevoir une prothèse et s'adapter parfaitement à elle. »

\section{LES OBJECTIFS}

Lobjectif initial était de créer un coussin amortisseur entre l'intrados prothétique et la muqueuse. Pour ce faire, la résine retard était changée régulièrement avant sa phase de dessiccation. Mais ce changement était réalisé sur un occluseur, porteur du modèle issu de l'empreinte secondaire. L'état de surface initial de la surface d'appui était ainsi conservé, il n’y avait donc aucune possibilité d'évolution de la surface d'appui. Joseph Lejoyeux étendit les objectifs initiaux en proposant une technique complète dite « technique directe » dont les buts étaient doubles :

I rendre possible l'évolution de la muqueuse des surfaces d'appui ;

$\checkmark$ modifier le comportement des muscles paraprothétiques, leur permettant d'améliorer la rétention des prothèses en favorisant l'extéroception de la muqueuse buccale et la proprioception linguale.

Pour cela, les résines à prise retardée étaient utilisées directement dans la cavité buccale d'où le terme de technique directe qui, contrairement à la technique indirecte, autorise l'évolution des caractéristiques macroscopiques et microscopiques de la surface d'appui.

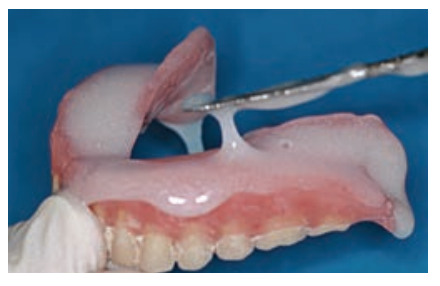

$\Delta$ Fig. 1.

Aspect du matériau en phase Gel, alors qu'il a déjà été placé dans la prothèse.

\section{LES MATÉRIAUX}

Les résines à prise retardée sont comme leur nom l'indique des résines PMMA dont la prise est retardée, contrairement aux résines traditionnelles chémoou thermopolymérisables.

Les résines à prises retardées se composent [5]:

I d'une poudre constituée de micro-billes de polyéthyl méthacrylate et de polyéthylène méthyl méthacrylate de diamètres différents ;

I d'un liquide mélange d'alcool éthylique et de plastifiants comprenant différents types de phtalates.

Le diamètre des micro-billes, leur composition, les proportions d'esters, d'alcool éthylique et/ou méthylique varient selon les fabricants. Ces variations expliquent les différences de comportement entre les produits, en particulier au niveau de leurs phases physiques [6].

\section{Les phases physiques de la réaction de prise}

Le mélange est le plus souvent préparé dans un rapport de deux volumes de poudre pour un volume de liquide. La réaction de prise se déroule en quatre phases successives, communes à tous les produits mais dont les caractéristiques et les durées varient selon les marques.

\section{$1^{\text {er }}$ temps : phase " Gel »}

Elle correspond à la phase de mélange des deux composants. Sa durée est d'environ 2 minutes. Mais le matériau doit être placé dans la prothèse au bout d'une minute donc toujours dans sa phase Gel (fig. 1) [7].

\section{$2^{\mathrm{e}}$ temps : phase «Plastique»}

Le gel commence sa polymérisation devenant plastique. Il se déforme pour s'adapter à une surface existante. C'est à ce stade que la prothèse est insérée dans la cavité buccale, puis placée sur la surface d'appui. La résine à prise retardée se modèle sous l'effet des pressions, qu'elles soient exercées par le praticien lors de l'insertion ou par le patient lors des fonctions physiologiques telles que la déglutition. C'est la durée de cette phase qui différencie le plus les différentes marques de résines retard $[8,9]$.

\section{$3^{\mathrm{e}}$ temps : phase «Élastique»}

Le matériau est souple, élastique mais ne se déforme plus sous l'effet des contraintes occlusales. Il agit en tant que coussin amortisseur.

\section{$4^{\mathrm{e}}$ temps : phase «Dessiccation »}

Le matériau perd ses propriétés élastiques, devient granuleux. Cette transformation est provoquée par la fuite des esters alcooliques vers le milieu salivaire alors que la salive pénètre dans la résine. La résine 
retard devient rugueuse, agressive vis-à-vis des tissus sous-jacents.

\section{POURQUOI RÉALISER LA MISE EN CONDITION TISSULAIRE ?}

Comme cela a été rappelé au début de cet article, l'objectif était de créer une base souple pour d'une part créer un effet de massage des tissus muqueux sous-jacents et d'autre part de répartir les forces fonctionnelles sur la surface d'appui. Cette approche a très rapidement disparu au bénéfice de la recherche de l'évolution de la surface d'appui muqueuse et de la modification du comportement des muscles périphériques.

La muqueuse de la surface d'appui peut être écrasée, blessée de manière plus ou moins généralisée par la prothèse existante. Les résines à prise retardée permettent de rétablir l'homéostasie de ces structures muqueuses sans pour autant imposer au patient le non-port de sa prothèse.

Les muscles paraprothétiques (lèvres-joues-langue) s'efforcent de maintenir les prothèses existantes mal conçues sur leurs surfaces d'appui respectives. Pour cela, des parafonctions s'installent telle que la position haute et postérieure de la langue qui maintient une prothèse maxillaire non rétentive. La modification des prothèses via l'utilisation des résines à prise retardée augmente la rétention, ce qui s'accompagne de la modification du comportement musculaire des muscles linguaux, labiaux, jugaux.

\section{QUAND RÉALISER LA MISE EN CONDITION TISSULAIRE ?}

La restauration de l'intégrité tissulaire des surfaces d'appui peut être théoriquement abordée dans les trois situations cliniques suivantes [10].

\Au stade pré-prothétique : très souvent, un patient se présente à la consultation avec des prothèses inadaptées, avec peut-être l'intention de les refaire.

\footnotetext{
Mais attention Danger!

Bien que très souvent utilisée, la mise en place de résine à prise retardée est, à ce stade, totalement contre-indiquée. En effet, la mise en place de résine à prise retardée dans la prothèse du patient est un acte irréversible. Si, pour une quelconque raison, le patient ne souhaite plus poursuivre les soins, il est en droit de demander qu'on lui restitue sa prothèse dans son état initial ce qui est impossible.

En conséquence, le praticien doit être conscient que : la mise en place de résine retard dans un intrados peut faire l'objet de plainte de la part du patient, surtout si le consentement éclairé n’a pas été dûment rempli, signé, après que le patient ait reçu une information exhaustive.
}

$\checkmark$ Au stade post-prothétique : en présence de prothèses anciennes inadaptées, malgré l'état des surfaces d'appui, le praticien peut avoir fait le choix d'élaborer de nouvelles prothèses. Dans un deuxième temps, les résines retardées seront utilisées pour traiter les tissus de la surface d'appui et parfaire la prothèse nouvellement réalisée.

I En présence de prothèses anciennes de bonne facture : elles ont été bien réalisées, les éléments prothétiques essentiels sont respectés orientation et niveau du plan occlusal, montage esthétique, montage fonctionnel seule l'adaptation aux surface d'appui est défectueuse. Cette solution thérapeutique est tout particulièrement indiquée chez les patients porteurs de prothèses anciennes auxquelles ils se sont tant sur le plan fonctionnel que psychologique parfaitement adaptés.

\section{COMMENT RÉALISER LA MISE EN CONDITION TISSULAIRE ?}

Les temps ou séquences de réalisation sont identiques quelle que soit la situation clinique.

La thérapeutique s'étale en moyenne sur cinq rendez-vous.

\section{$7^{\text {re }}$ séance}

La prothèse existante est corrigée, les surextentions supprimées, les bords trop longs raccourcis. Les zones muqueuses douloureuses sont déchargées dans l'intrados. L'utilisation de silicone fluide déposé dans l'intrados de la prothèse qui est replacée avec pression sur la surface d'appui permet de révéler facilement les éventuelles zones de surpression (fig. 2).

Aux retouches réalisées s'ajoutent trois corrections systématiques : décharges au niveau des lignes mylo-hyoïdiennes ou lignes obliques internes, au niveau des trigones rétromolaires et du joint sub-lingual (fig. $3 \mathbf{a}-\mathbf{b}-\mathbf{c})$. Enfin, l'intrados est légèrement gratté sur toute sa surface puis nettoyé à l'aide de chloroforme ou d'éther.

Le matériau choisi est préparé selon les prescriptions du fabricant, généralement un rapport 2 poudre 1 liquide. Notre choix se porte sur l'Hydro-Cast ${ }^{\circledR}$ ou le FITT $^{\circledR}$, en raison de leurs temps de gélification et de phase plastique relativement courts.

Le matériau est étendu dans l'intrados au stade de gel puis inséré dans la cavité buccale alors qu'il amorce

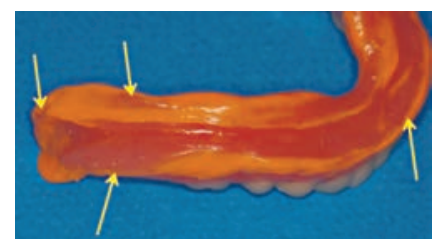

4 Fig. 2.

Le silicone révèle les surpressions et sur-extentions de la base prothétique. 

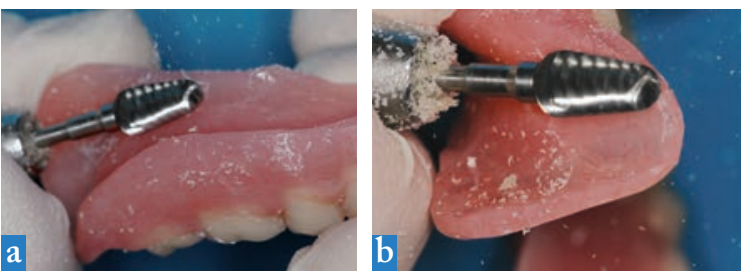

$\Delta$ Fig. 3.

Retouches systématiques au niveau du trigone (a), de la ligne mylo-hyoïdienne (b), du join sub-lingual (c).
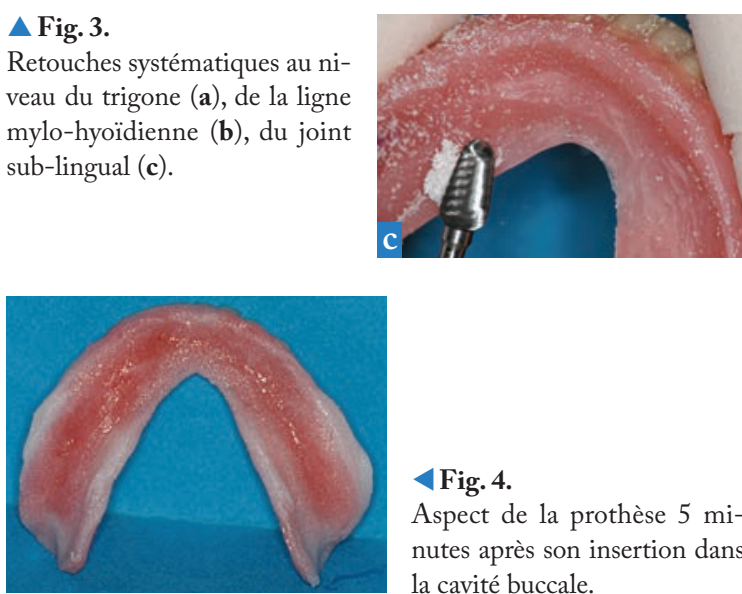

$<$ Fig. 4.

Aspect de la prothèse $5 \mathrm{mi}^{-}$ nutes après son insertion dans la cavité buccale.
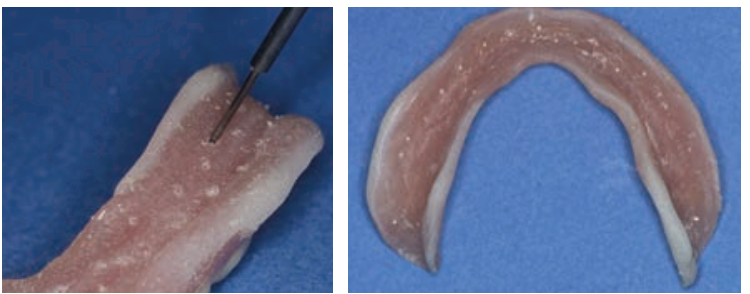

$\Delta$ Fig. 5.

Lintrados est creusé sur une profondeur de $2 \mathrm{~mm}$. Une fraise calibrée sert de jauge de profondeur. (a) Résultat final. Noter la présence des bords en résine à prise retardée $(\mathbf{b})$.

son passage de la phase Gel à la phase Plastique. Le patient est guidé en occlusion d'intercuspidie maximale, les prothèses entrant en contact avec une très légère pression pour éviter de chasser le matériau de l'intrados sans pour autant augmenter la dimension verticale de l'occlusion. Après quelques instants, le patient est invité à mobiliser ses joues, sa langue afin d'éviter toute surextension. La prothèse est désinsérée à la fin de la phase Plastique soit après 5 à 6 minutes avec le FITT $^{\circledR}$ ou l'Hydro-Cast ${ }^{\circledR}$ mais 16 minutes pour le Coe-Confort ${ }^{\circledR}$ ou le Visco-Gel ${ }^{\circledR}[11]$ (fig. 4). La prothèse est retirée, examinée, dans l'idéal, la résine retard s'étend sur toute la surface ne laissant pas apparaître la résine de la base.

À la fin de cette séance, les conseils suivants sont prodigués au patient :

Ine pas consommer d'aliments qui laissent des grains

(framboises, figues, fraises, etc.) ;

Inettoyer l'appareil en le passant sous l'eau ;

Ine pas brosser la prothèse ;

Ine pas poser la prothèse sur des papiers type « Kleenex »;

Ine pas utiliser des produits d'hygiène qui dans leur majorité détruisent les résines retard (tableau 1). $\checkmark$ Tableau 1. Incompatibilités entre certains couples résinesproduits de nettoyage [12].

\begin{tabular}{|c|c|c|}
\hline Résine retard & Agents chimiques & Nom commercial \\
\hline FITT $^{\circ}$ & Peroxydes & Steradent \\
\hline Soft-Liner
\end{tabular}

\section{$2^{\mathrm{e}}$ séance}

Dans l'idéal, le patient est revu 48 heures après, 72 heures au maximum. Le patient est invité à exprimer les problèmes, les difficultés ressenties, les améliorations constatées. Elles se regroupent le plus souvent en deux types de doléances : problèmes d'instabilité de la prothèse ou de douleurs au niveau de la surface d'appui.

L'approche thérapeutique se déroule en deux temps : contrôle de l'occlusion, contrôle de la résine retard :

$\checkmark$ contrôle de l'occlusion : dans un premier temps, les contacts occlusaux sont examinés (papier à articuler), les éventuels contacts prématurés sont corrigés. Cela permet de répartir les charges occlusales de manière harmonieuse sur la surface d'appui ;

I contrôles de la résine retard : l'intrados, les bords de la prothèse sont contrôlés. Des irritations, des blessures sont recherchées au niveau de la surface d'appui. Des corrections sont effectuées en respectant le principe suivant : quelles que soient les corrections faites (bords-intrados), la remise en place de la prothèse sur la surface d'appui doit se faire de manière précise et sûre afin de ne pas perdre les relations occlusales (DVO-OIM).

Pour cela, le praticien aura trois approches :

I si la surface d'appui est imparfaite, les bords sont conservés. L'intrados est creusé fortement sur une profondeur de $2 \mathrm{~mm}$, puis garni, sans excès, de résine à prise retardée (fig. 5). Lélasticité recherchée nécessite en effet une épaisseur de matériau d'environ $2 \mathrm{~mm}$;

I si les bords sont imprécis, la prothèse est instable. Dans ce cas, l'intrados est conservé, les bords raccourcis, puis à nouveau regarnis de résine retard ;

I si la situation est mixte, les corrections sont effectuées mais en ayant soin de ne jamais perdre l'adaptation de la prothèse sur la surface d'appui.

Les corrections effectuées, les zones retouchées sont regarnies de la résine retard utilisée. La prothèse est réinsérée avec précision sur la surface d'appui grâce aux contrôles que fournissent les bords ou l'intrados. Le patient est guidé en occlusion, les contacts s'effectuant de manière très légère, afin de ne pas chasser la résine.

Après gélification, la prothèse est retirée de la cavité buccale, la résine retard examinée, les excès éliminés. Dans les zones où la résine de la base apparaît, des corrections adjonctions sont pratiquées. 

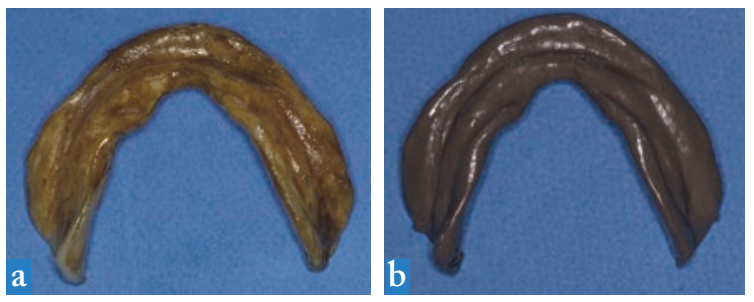

A Fig. 6.

Mise en place de l'adhésif (a). Empreinte de la surface d'appui (b).

Lorsque l'ensemble des corrections est terminé, la surface de la résine est enduite de Microseal ${ }^{\circledR}$ (KAY See dental) ou de J-305 monopoly syrup (Factor II, Inc., Lakeside AZ). Cet enduit est ensuite polymérisé à l'aide d'une simple lampe.

Ces produits permettent de maintenir les qualités élastiques des résines en s'opposant aux échanges entre les esters alcooliques de la résine et la salive.

\section{$3^{\mathrm{e}}$ séance}

Quarante-huit heures après, cette séance se déroule selon la même chronologie que la précédente : prise en compte des doléances, examen des bords de l'intrados, etc.

Cette méthodologie se répète jusqu'à ce que l'intégrité tissulaire de la surface d'appui soit restaurée, les qualités mécaniques rétablies. Généralement, la séquence de quatre rendez-vous décrite est suffisante.

\section{Avant-dernière séance}

Le praticien s'enquiert auprès du patient des sensations qu'il a éprouvées : gênes, douleurs, inconforts, instabilité. Si les réponses sont défavorables, la prothèse est examinée, corrigée selon les mêmes séquences que celles précédemment utilisées.

Si la réponse est favorable, la prothèse est retirée de la cavité buccale. Une empreinte de surfaçage est réalisée à l'aide de polysulfure (Permlastic Light ${ }^{\circledR}$ ). Pour cela, l'intrados est nettoyé (alcool) puis enduit de l'adhésif adapté (fig. 6). Il est préférable d'utiliser une colle très liquide afin d'avoir une épaisseur minimale (adhésif dilué avec de l'acétone). Après garnissage de l'intrados, l'empreinte est prise bouche fermée en occlusion. L'empreinte est contrôlée puis adressée au laboratoire pour une réfection de la base prothétique. La réfection consiste dans le changement complet de la base par une nouvelle résine thermopolymérisable. Cette étape doit être réalisée dans les délais les plus brefs, 24 heures dans l'idéal.

\section{Dernière séance}

La réfection faite, la prothèse avec sa nouvelle base est réinsérée. Dans la mesure où la phase de laboratoire a été conduite avec précision, l'insertion ne présente aucune difficulté.

\section{CONCLUSION}

En prothèse amovible totale, l'utilisation des résines à prise retardée demeure d'actualité. Car cette approche thérapeutique, bien que décrite depuis les années 1960, garde toute sa valeur. Bien conduite, elle permet de répondre à des situations cliniques bien précises en particulier :

$\checkmark$ l'amélioration d'une prothèse récemment réalisée ; Ila réfection de prothèses anciennes bien conçues auxquelles les patients sont parfaitement adaptés, en particulier chez les patients âgés.

En revanche, ces techniques ne devront jamais être utilisées ou avec beaucoup de prudence en présence de prothèses anciennes inadaptées. La mise en place de ces résines dans l'intrados d'une prothèse pour « dépanner » le patient peut être à l'origine de conflits juridiques, éventualité dont le praticien doit être parfaitement conscient.

\section{Bibliographie}

[1] Lytle R. Management of abused tissues in complete denture construction. J Prosthet Dent 1957;7:27-42.

[2] Chase W. Tissue conditioning utilizing Dynamic Adaptive Stress. J Prosthet Dent 1961;11:804-15.

[3] Pound E. Preparatory dentures: A protective philosophy. J Prosthet Dent 1965;15:5-18.

[4] Lejoyeux J. La mise en condition tissulaire. RFOS 1962.
[5] Braden M. Tissue conditioners. I. Composition and structure. J Dent Res 1970;49(1):145-8.

[6] Murata H, Hamada T, Taguchi N, Shigeto $\mathrm{N}$, Nikawa $\mathrm{H}$. Viscoelastic properties of tissue conditioners--influence of molecular weight of polymer powders and powder/liquid ratio and the clinical implications.J Oral Rehabil 1998;25(8):621-9.

[7] Murata H, Chimori H, Hamada T, McCabe JF. Viscoelasticity of dental tissue conditioners during the sol-gel transition. J Dent Res 2005;84(4):376-81.

[8] Braden M. Tissue conditioners:

II. Rheologic properties. J Dent Res 1970;49(3):496-501.
[9] Murata H, Hamada T, Djulaeha E, Nikawa H. Rheology of tissue conditioners. J Prosthet Dent 1998;79(2):188-99.

[10] Hue O, Berteretche M-V. Prothèse complète : réalité clinique, solutions thérapeutiques. Quintessence international. Paris, 2002.

[11] Murata H, Iwanaga H, Shigeto N, Hamada T. Initial flow of tissue conditioners-influence of composition and structure on gelation. J Oral Rehabil 1993;20(2):177-87.

[12] Murata H, Chimori H, Hong G, Hamada T, Nikawa H. Compatibility of tissue conditioners and denture cleansers: influence on surface conditions. Dent Mater J 2010;29(4):446-53. 